\title{
A Method for Engineering Design Selection of Complex Systems Based On System Engineering
}

\author{
Jia $\mathrm{Li}^{1, \mathrm{a}}{ }^{*}$ and Fayuan $\mathrm{Wei}^{1, \mathrm{~b}}$ \\ ${ }^{1}$ Institute of Systems Engineering at China Academy of Engineering Physics, \\ Mianyang, China \\ alijia@caep.cn \\ bweify@caep.cn
}

Keywords: Conceptual Design, Decision Making, System Engineering.

Abstract. The modern complicated large engineering is significant to the nation and society. The initial conceptual design selection could decide most of the whole engineering project. It's a multi-objective, multi-criteria, multi-field process. Basing experts' experience, the traditional plan selection depends largely on qualitative information and is subject to subjective factors. The modern decision making methods can help the process more quantitative and objective. Using System Engineering (SE) methodology combined qualitative and quantitative information, this paper discusses a comprehensive and concise method about the complex system design selection and decision making. The weight and metrics are discussed as the important impact during objective break-down, quantitative modeling and accessing. expert knowledge of complicated engineering project is conducted, in order to support a more objective, science-based decision making during conceptual design phase of large engineering system design.

\section{Introduction}

With the developing of society, the activity of human changing the world become more and more complicated. Many large complicated engineering come up, such as aviation, aerospace, shipping, transportation, hydraulic engineering, etc. The modern engineering is significant to the nation and society. It's complicated, difficult, long term, large investment, and involving many departments. The overall design should aim the total goal in the holistic point of view.

During the early lifecycle conceptual design, the design selection and decision making are crucial to the engineering project because they decide the costs, process, future performance and so on. Meanwhile, it has the difficulties about multi-objective, multi-criteria, multi-fields. It also be affected from lacking of the precise information and some uncertainties in the future [1].

In the traditional engineering practice, a lot of empirical methods, such as symposiums with experts, qualitative judgments, etc., are used to get the approximate solutions for plan decision making. This kind of decision making is feasible, flexible and has some rationality. However, facing the more and more complicated modern engineering, the traditional method cannot challenge the interdisciplinary, multi-field, complicated relationships among systems and subsystems. Besides, owing to multiple of departments, personnel and steps, the plan shall be greatly affected from different positions and persons. The final result may be large deviation from the initial goal, owing to the great subjectivity of the empirical methods. In addition, because the qualitative, empirical processes and conclusions cannot be represented, recorded and managed explicitly and precisely by the modern computer aided systems, the knowledge is difficult to be inherited and reused in the future [2].

Nowadays, the Systems Engineering (SE) theory derives from the empiricism-based way to solve the engineering application problems. SE theory and methodology belongs to the category of engineering science, basing engineering science concept and its characteristics, interdisciplinary, systemic interrelated, science and experience combined [2, 3]. From the late of the 20th century, it impacts on the large engineering practices significantly, when the SE theory comes into being and be implemented. Aiming the total goal, the SE theory emphasizes the integrality, relationships, 
hiberarchy, and environmental adaptability. It's an important academic guidance for the development and construction of the large, complicated engineering design.

Aiming the large, complicated engineering, which is interdisciplinary, multi-fields, application of both experience knowledge and scientific theories, with qualitative and quantitative knowledge, this paper discusses a comprehensive decision making method with the SE theory. It focuses on the objective analysis, the metrics and criteria construction and the weights allocation, which would affect the result directly. The method is also combined with the qualitative experience of experts and quantified parameters.

\section{Common methods of plan selection and decision making}

During the initial conceptual design phase, the whole engineering system should be analyzed in the holistic point of view, the candidate plans should be assessed comprehensively, then the decisions would be made. Generally, the plan selection and decision making process include three phases: objective analysis, assess modeling with the metrics and criteria, calculation and assessment. Fig. 1 describes the main steps. After the requirement definition and objective analysis, the sub-objectives and functions are abstracted. With the basic principles, some hypothesis and solutions are found out, which would make up of the candidate plans by different combination of functions and structures. At the same time, the group of key performance index about plans can compose the metrics and criteria for assess modeling. It would get the selection result after inputting the different candidate plans via the assessment model. $[4,5]$

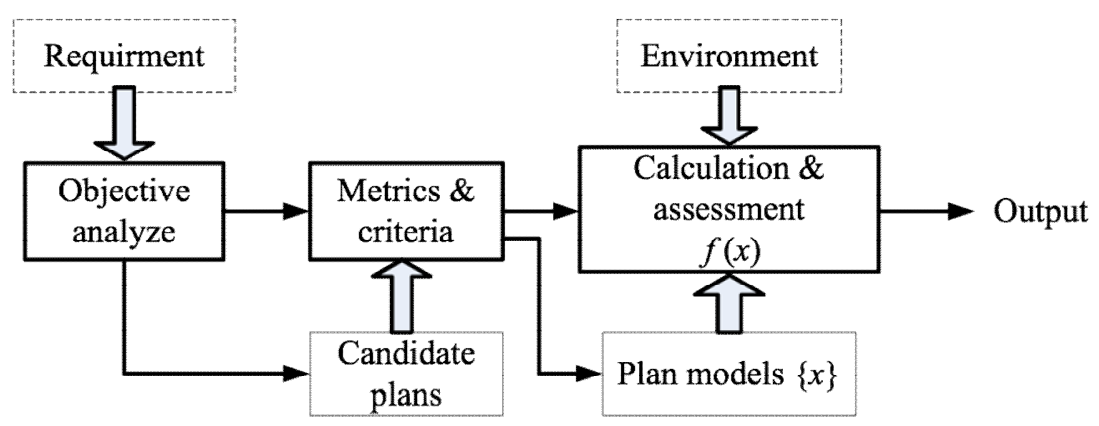

Fig. 1 the Process of Plan Selection and Decision Making

Table 1 the Common Methods for Decision Making

\begin{tabular}{|c|c|}
\hline Methods & Characteristics \\
\hline Experts Evaluation & $\begin{array}{l}\text { To get the whole evaluation by experts review, discuss, give grate or } \\
\text { rank, etc. }\end{array}$ \\
\hline $\begin{array}{l}\text { Analytical Hierarchy } \\
\text { Process (AHP) }\end{array}$ & $\begin{array}{l}\text { To construct the judgment matrix via pared-compare and calculate the } \\
\text { priority of plans. }\end{array}$ \\
\hline $\begin{array}{l}\text { Quality Function } \\
\text { Deployment (QFD) }\end{array}$ & To breakdown the function with customer requirements driven \\
\hline $\begin{array}{l}\text { Multi-objective Decision } \\
\text { Making, (MODM) }\end{array}$ & $\begin{array}{l}\text { To combine the multi-objectives into an optimized single objective to } \\
\text { assess the system in the whole. }\end{array}$ \\
\hline $\begin{array}{l}\text { Fuzzy Comprehensive } \\
\text { Evaluation (FCE) }\end{array}$ & To estimate the multi-factors using fuzzy mathematics methods. \\
\hline $\begin{array}{l}\text { Grey Theory Based } \\
\text { Decision }\end{array}$ & $\begin{array}{l}\text { Aiming the grey systems which have only parts of information } \\
\text { known. }\end{array}$ \\
\hline Case-Based Reasoning & $\begin{array}{l}\text { To gain the similar case solutions via analogy and association basing } \\
\text { cases reasoning. }\end{array}$ \\
\hline Technology Frontier & $\begin{array}{l}\text { To construct the ideal solution with the balancing of the Performance } \\
\text { Effectiveness and Economics Effectiveness. To assess the plan by the } \\
\text { distance from the ideal point. }\end{array}$ \\
\hline
\end{tabular}


Table 1 sums up the common methods for plan selection and decision making. They are aiming different requirements and objects, using different metrics and criteria, constructing different mathematics models to calculate and assess $[4,5,6,7,8]$.

During the early lifecycle conceptual design, the full-scale view, macro analysis and comprehensive understanding are very needed. Thus, most of the decision making methods include the experts experience. To assess the plan, the requirements and goals decide the characteristics of the plan which the assess mathematical model depends on. Meanwhile, the weight of each index impacts the results of assessment directly. Thus, it's important to focus on the assess mathematical modeling and weight allocation. Besides, owing to the complexity of the large engineering, the more simple and concise methods are more feasible in the engineering practices.

\section{A comprehensive method for decision making}

Aiming the large complicated engineering systems, this paper discusses a comprehensive concise method for the plan selection and decision making, which combines several common methods and follows the main approach as Fig. 1 shows. Begin with the requirement, it includes three steps: objective analysis, assess modeling, calculation and assessment. The metrics, criteria and each index weight are focused owing to their directly impacts on the assess result.

Objective analysis. Objective analysis is the process to define the requirement and break down the total goal into a series of evaluation items or key performance index, level by level. The objective classifying is important because that different kinds of objectives use different criteria. The objective break down reifies or concretes the objectives and makes clear the relationships among the key performance index. The whole process of objective analysis is the base to build the evaluation framework and assessment model.

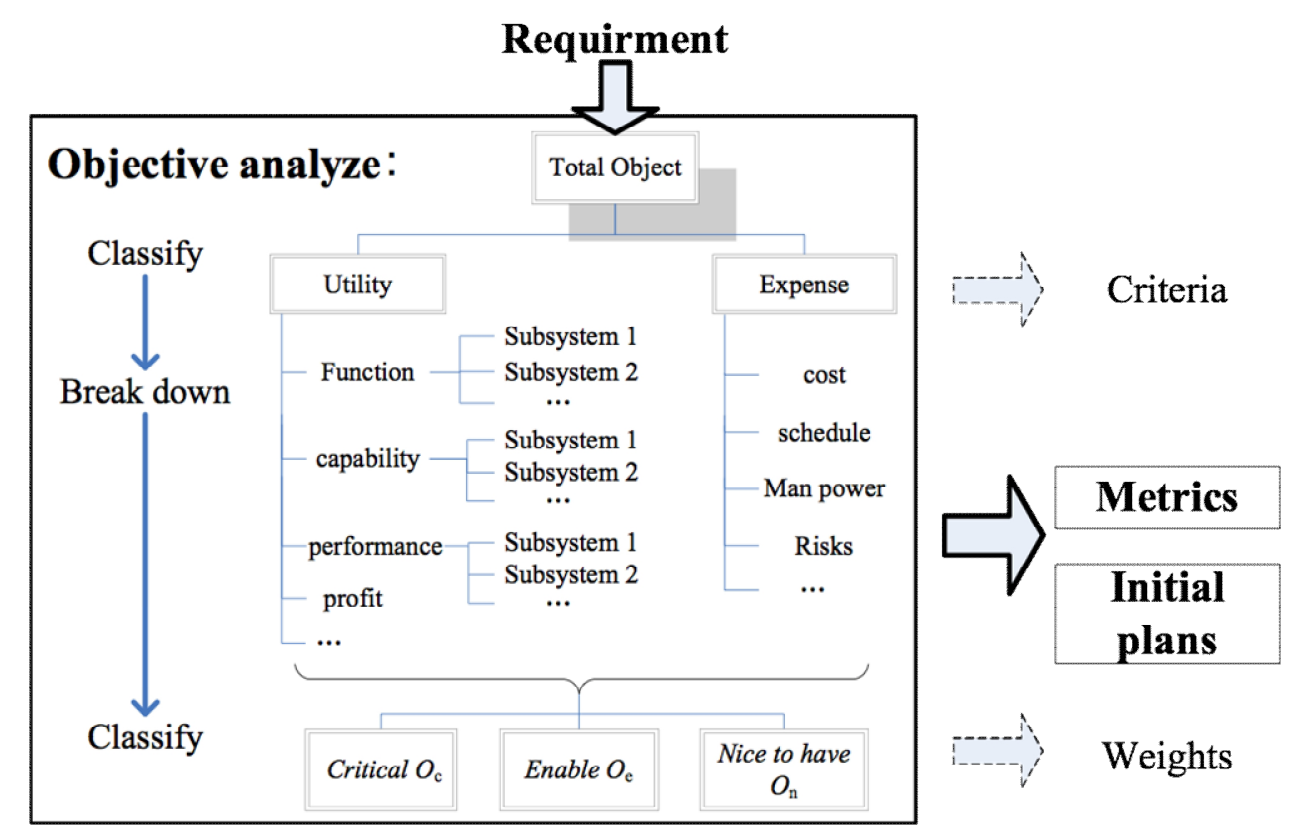

Fig. 2 the Objective Analyze Framework

Generally, the requirement is a kind of macro and qualitative goal. In consideration of different requirements, firstly, the macro goal can divided into two categories, the utilities and expensive with two different criteria. The utility objectives are the bigger the better and expect the optimum performance and the maximum value, on the contrary, the expensive objectives expecting the optimum economics and the minimum value. However, it's impossible to gain the both maxima and minima, balance and optimize are needed $[4,8]$.

Secondly, breakdown the objectives by reifying the both categories. For example, the utility can be concreted as functions, performance, profile, etc.. The expense can be concreted as investment, cost, schedule, man power, risk, etc.. During this, some of the objectives are extracted as a series of 
quantitative index, such as cost, schedule, profile, etc.. But lots of the rest are qualitative. Breaking down from the system level to sub-system level, more and more quantitative index such as sub-functions and parameters, are defined.

At the same time, by evaluating the importance of the objectives, it can be divided into three gradations: critical objectives $\left(\mathrm{O}_{\mathrm{C}}\right)$, enabling objectives $\left(\mathrm{O}_{\mathrm{E}}\right)$ and nice-to-have objectives $\left(\mathrm{O}_{\mathrm{N}}\right)$. The critical grade means it's crucial to success and must be achieved. The enabling grade means it's very helpful to achieve the critical goals. The nice-to-have grade is the icing on the cake and might make the endeavors easier.

Fig. 2 describes the procedure of the objective analyze method. It's combined with the objective classifying and breaking down to get the sub-objectives and the assess index.

Assess modeling.According to the objectives, the candidate plans are promoted by empirical hypothesis by the experts or teams. These design conceptions are almost vague, inaccurate and qualitative. Then, considering the external environment, each plan models can be constructed by the group of crucial functions, parameters, relationships and influential factors each corresponding to the sub-objectives and index which gained in the first step. The models consist of quantitative parameters and qualitative effects.

Table 2 the Judgment Matrix and the Eigenvectors

\begin{tabular}{c|cccc|c}
\hline A & E1 & E2 & $\ldots$ & Ej & W \\
\hline E1 & E11 & E12 & $\ldots$ & E1j & W1 \\
E2 & E21 & E22 & $\ldots$ & E2j & W2 \\
$\ldots$ & $\ldots$ & $\ldots$ & $\ldots$ & $\ldots$ & $\ldots$ \\
Ei & Ei1 & Ei2 & $\ldots$ & Eij & Wi \\
\hline
\end{tabular}

According to the plan model framework, the assess mathematical model $\mathrm{f}(\mathrm{x})$ can be constructed with the series of assess index and its corresponding criteria.

In this assess model, the weight of each index should be allocated reasonably because of its directly impact on the final result. With the SE theory, the Analytical Hierarchy Process (AHP) [1] is widely used to allocate the index weights, combined with the experts experience. We use AHP method to construct the judgment matrix to calculate each index weight with experts suggestions and judgments.

Firstly, the importance of each sub-objectives or each influential factors are paired-compared in the judgment matrix A. In Eq. 1, Eij is the importance comparison between Ei and Ej with the proportional scale from 1 to 9 giving by experts. Secondly, $\mathrm{W}$ is the eigenvector matrix of matrix A, which represents the quantitative importance of factor Ei in Eq. 2. Lastly, the coherence of the judgment matrix must be verified. Table 2 shows an example about the factor importance comparison.

$$
\begin{aligned}
& A=[E i j], i, j=1,2, \ldots, n . \\
& W=[W i], j=1,2, \ldots, n .
\end{aligned}
$$

Calculation and assessment. There are two parts included in this step, the calculation of each plan's comprehensive quantitative performance with the assess mathematical model and the final result review by the experts or teams, which makes the result more reasonable combining the both quantitative and qualitative factors.

At first, corresponding to each factors, the performance comparison among candidate plans are in the judgment matrix $P$, where the qualitative factors are valued by expert marking and the quantitative factors are normalized. In Eq. 3, i represents the number of candidate plans, j represents the number of factors. In Eq. 4, Wp is the total weight value, and Wpi represents each plan's advantage.

$$
P=[P i j], i, j=1,2, \ldots, n \text {. }
$$

错误！未找到引用源。 $\mathrm{W} \cdot \mathrm{P}=\mathrm{Wp}$.

After experts assessing or reviewing the quantified advantages of each plan, the better plan is selected out. The result is qualitative and quantitative combined and more reasonable. 


\section{Application Example}

There is an example about one engineering project's plan selection and decision making during the conceptual design phase. Table 3 shows the assess metrics and criteria with the objective analysis method.

Table 3 Objective Breaking Down and Analysis

\begin{tabular}{cccc}
\hline Total goal & Categories/criteria & Index & Sub-objective classes \\
\hline & & Crucial functions & Critical \\
& & Additional functions & Nice-to-have \\
& & Efficiency & Critical \\
& Utility/ Maximum & Reliability & Critical \\
Comprehensive & & Safety & Critical \\
requirement of & & Technical maturity & Critical \\
the system & & Standardized rate & Critical \\
& & Maintainability & Enabling \\
& & Technology advancement & Enabling \\
& & Innovation \& prospective & Enabling \\
& & Cost & Nice-to-have \\
& & Schedule & Enabling \\
& Expensive & Weight & Critical \\
& /Minimum & Size & Enabling \\
& & & Enabling \\
\hline
\end{tabular}

Table 4 the Conceptual Design Selection Assess Result

\begin{tabular}{|c|c|c|c|c|c|}
\hline \multirow{2}{*}{ Goal } & \multirow[t]{2}{*}{ Index } & \multirow[b]{2}{*}{ W } & \multicolumn{2}{|c|}{ Design Plan } & \multirow{2}{*}{ Note } \\
\hline & & & P1 & P2 & \\
\hline \multirow{7}{*}{$\begin{array}{c}\text { Critical } \\
\left(\mathrm{O}_{\mathrm{C}}\right)\end{array}$} & Crucial functions & 0.226 & 0.129 & 0.097 & Experts marked qualitatively \\
\hline & Schedule & 0.147 & 0.054 & 0.092 & The normalized data \\
\hline & Reliability & 0.100 & 0.063 & 0.037 & The normalized data \\
\hline & Safety & 0.088 & 0.038 & 0.050 & Experts marked qualitatively \\
\hline & Environmental adaptability & 0.086 & 0.049 & 0.037 & Experts marked qualitatively \\
\hline & Life & 0.039 & 0.015 & 0.023 & The normalized data \\
\hline & Efficiency & 0.025 & 0.013 & 0.012 & The normalized data \\
\hline \multirow{6}{*}{$\begin{array}{c}\text { Enabling } \\
\left(\mathrm{O}_{\mathrm{E}}\right)\end{array}$} & Cost & 0.098 & 0.033 & 0.065 & The normalized data \\
\hline & Maintainability & 0.046 & 0.032 & 0.014 & The normalized data \\
\hline & Technical maturity & 0.027 & 0.019 & 0.008 & The normalized data \\
\hline & Standardized rate & 0.026 & 0.018 & 0.008 & The normalized data \\
\hline & Size & 0.018 & 0.008 & 0.010 & The normalized data \\
\hline & Weight & 0.009 & 0.003 & 0.006 & The normalized data \\
\hline \multirow{3}{*}{$\begin{array}{c}\text { Nice-to- } \\
\text { have } \\
\left(\mathrm{O}_{\mathrm{N}}\right)\end{array}$} & Innovation \& prospective & 0.025 & 0.008 & 0.017 & Experts marked qualitatively \\
\hline & Additional functions & 0.020 & 0.009 & 0.011 & Experts marked qualitatively \\
\hline & Technology advancement & 0.020 & 0.005 & 0.015 & Experts marked qualitatively \\
\hline \multicolumn{3}{|c|}{ Comprehensive weight value: } & 0.496 & 0.504 & \\
\hline
\end{tabular}

Table 4 shows the assess result of the two design plans in the assess mathematical model. The assess index are identified and their importance and weight are compared in column $\mathrm{W}$, using the AHP method. The quantified performance value of two design plans are listed in column P1 and P2.

The calculated comprehensive weight value shows that the second is better. After the expert reviewing, it can make a decision on the engineering project's conceptual design selection. 


\section{Result}

Aiming the large, complicated engineering with the characteristics of interdisciplinary, multi-fields, application of both experience knowledge and scientific theories, this paper discusses a comprehensive and concise method about plan selection and decision making with the combination of some common methodologies, including the calculation and experts experience quantification. Basing the SE theory, this method focuses on and describes the procedure of the objective analysis, the metrics and criteria construction and the weights allocation, which affect the result greatly. An example demonstrates this method.

\section{Conclusions}

The application of the SE theory can improve the problem about qualitative and subjective decision making in the complex engineering system design and construction. It helps more objective, science-based decision making during the conceptual design phase.

However, during the early lifecycle of the engineering system design, the full-scale view, macro analysis and comprehensive understanding are very needed. Thus, not only the quantitative calculation but also the qualitative information and fuzzy method should be combined in the decision making, especially the experts experience.

In addition, it's very important to deal with the comparison of the influential factors. The weight value of each index importance is directly effects the result. There are many mathematical methods shall be compared and applied.

Furthermore, the candidate plan can be optimized according to the plan selection and assessment result with this method. The plan optimization basing this methodology will be discussed in the later.

Besides, owing to the complexity of the large engineering, the more simple and concise methods are more feasible in the engineering practices. Meanwhile, the decision making for a large engineering project relates greatly to the management level. In order to apply this kind of quantitative method on real decision making, there are lots of works need carry out, such as standardization and systematization.

\section{References}

[1] Zhou Huaren, 2011, System Engineering, Tsinghua university Publication, China.

[2] Li Jia, Wei Fayuan, 2014, "Knowledge Representation of Engineering Designs with the Concept of Engineering Science", X I International Science Conference, 2014 ICMAE, Geneva, Switzerland. 8(9) Part I, pp. 145-147.

[3] Qian Xuesen, 1948, "Engineering and Engineering Science", Journal of the Chinese Institution of Engineers 6(1948), pp. 1-14.

[4] Liu Xiaodong, Song Bifeng, 2004, "Theories and Methods for Decision Making in Complex Engineering System Conceptual Design: A Survey", Systems Engineering theory and practice, 12, pp. 72-77.

[5] Masataka Yoshimura, 2012, System Design Optimization for Product Manufacturing, China Machine press, China.

[6] Xiong Yunfeng, Chen Zhanglan, Yuan Hongli, 2013, "Research on the decision-making method of ship based on QFD”, Ship Science and Technology, 35(9), pp. 44-47.

[7] Cui Jianpeng, Xin Yongping, Liu Xiaojian, 2012, "Research on Surface to Air Missile Type Selection Based on Multi-criteria Gray Decision-making”, Tactical Missile Technology, 2012(1), pp.7-10.

[8] Michael A. Schaffner, Adam M. Ross, Donna H. Rhodes, 2014, “A Method for Selecting Affordable System Concept: A Case Application to Naval Ship Design”, Procedia Computer Science, 28(2014), pp. 304-313. 